\title{
An Improved Method of Geomagnetic Aided Inertial Navigation Algorithm with Gyro and Accelerometer Error Corrected Online
}

\author{
Song ZhongGuo ${ }^{1}$, Gao Jiuxiang ${ }^{1}$, Zhang Jinsheng ${ }^{2}$, and Xi Xiaoli ${ }^{1}$ \\ ${ }^{1}$ Faculty of Automation and Information Engineering, Xi'an University of Technology, Xi'an, China \\ ${ }^{2}$ Xi'an research inst of hi-tech, Xi'an, China
}

\begin{abstract}
In consideration of the problem that traditional geomagnetic aided navigation method cannot reduce the scaling error of indication track in inertial navigation system (INS), which will further limit the error correction precision of gyro and accelerometer, an improved geomagnetic matching algorithm based on affine transformation is proposed in this paper. A geomagnetic matching algorithm led to the optimal affine transformation solution by Procrustes analysis is presented and develops latitude and longitude reference information. Then a 13-dimensionalstate extended Kalman filter which estimates the attitude misalignment angles, the position error, the velocity error, the Gyro drift, and accelerometer error is introduced to continuously update the output of INS and remove the accumulative error. The results show that geomagnetic aided navigation based on improved algorithm has better location accuracy and correction accuracy of INS than the traditional method.
\end{abstract}

\section{Introduction}

The orientation and velocity error of pure Inertial Navigation System (INS) will accumulate and result in divergence over time due to the existence of gyro drift and accelerometer deviation, which will further affect the positioning precision. Geomagnetic aided navigation system (GANS) utilizes the matching algorithm to compare geomagnetic field profile acquired real-time with the reference geomagnetic map stored on board for matching. The matching results will be utilized to correct gyro drift and accelerometer error of INS. GANS has characteristics of strong autonomous, good concealment and working consistently over earth surface without reliance on external radio navigation signals. Besides, the navigation errors of GANS don't cumulate over time [1]. Due to these advantages, GANS has essential application value in underwater vehicle, unmanned aerial vehicle and indoor pedestrian navigation in recent years [2].

In the 1960s, American E-System Corporation proposed contour-based magnetic matching MAGCOM system [1]. Then magnetic navigation technology has a rapidly developed in various countries from then on. The simulation platform of GANS was established and validated the iterated closest contour point (ICCP) matching algorithm better than traditional contour matching algorithm [3]. Simulation of geomagnetic/inertial integrated navigation System was presented by Zhou [4], which validated a new geomagnetic matching algorithm based on MSD algorithm and ICCP algorithm is effective. The SITAN algorithm makes use of terrain elevation information in
GANS and achieves reliable results [5]. Literature [6] presents a locating method of geomagnetic/inertial integrated navigation system to improve the accuracy of initial geomagnetic matching position.

In order to solve the problem that ICCP has a high requirement no scaling on the INS indication track and enhance the accumulative error compensation accuracy and onboard performance of INS, this paper designed an information fusion filter with the combination of the improved ICCP described in [7]. The paper is organized as follows. Section 2 addresses the principle of GANS, Section 3 is devoted to key technologies of GANS, Section 4 presents the simulation and the results. Conclusions are given in Section 5.

\section{Principle of GANS}

The process of the GANS is shown in Figure 1. The navigation processor is mainly composed of matching algorithm and information fusion filter. The geomagnetic reference maps along the track are stored in the vehicle firstly. There are two primary inputs, one is the geomagnetic intensity processed from three-axis fluxgate magnetometers, and the other is the indication track collected from INS. During the vehicle cruise along the track, magnetometers are detecting background geomagnetic field to form the real-time magnetic sequence, which will be compared with the stored map to determine the difference and position information about the vehicle with matching algorithm. Then EKF estimates 
error parameters to correct INS and continuously update navigation information.

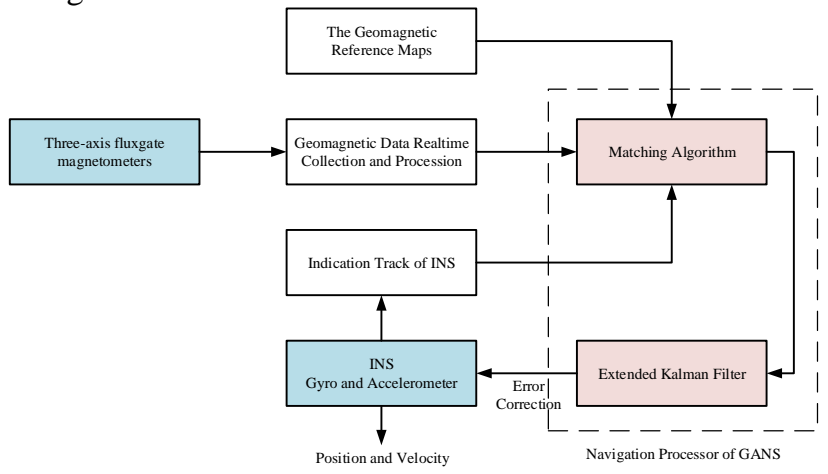

Figure 1. The process of the GANS

\section{The Key Technology of GANS}

\subsection{Matching algorithm}

The iterated closest contour point (ICCP) algorithm is a kind of matching algorithm based on contour constraint. It was derived from the iterative closest point (ICP) algorithm of image registration and often used in terrain matching and gravity matching navigation [8].

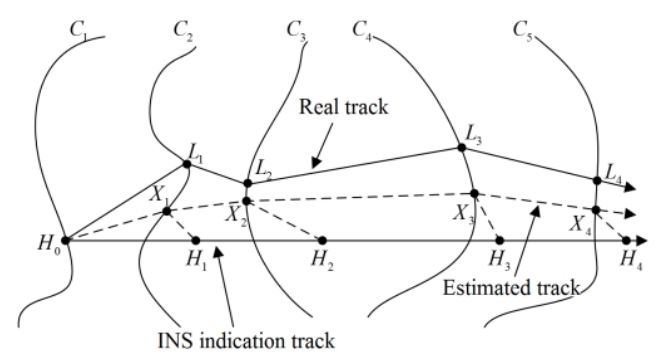

Figure 2. Illustration of INS indication track, estimated track, and real track.

The principle of ICCP algorithm is shown in Figure 2. Let us denote the INS indication track by $\left\{H_{i}\right\}(i=1,2, \mathrm{~L}, N)$ ( $\mathrm{N}$ is the number of points for one matching), which is deviant from the real track $\left\{L_{i}\right\}(i=1,2, \mathrm{~L}, N)$ due to measurement errors and drifts. The magnetometer provides the corresponding measured geomagnetic intensity $\left\{g_{i}\right\} \quad(i=1,2, \mathrm{~L}, N)$, and the corresponding geomagnetic field contour $\left\{C_{i}\right\} \quad(i=1,2, \mathrm{~L}, N)$ can be searched out through geomagnetic values $g_{i}$ from the pre-stored geomagnetic map. After which, the estimated track $\left\{X_{i}\right\}(i=1,2, \mathrm{~L}, N)$ can be obtained from $C_{i}$, which is the matching target. The navigational errors can be corrected by a rigid transformation of the indicated track into the real track. The rigid transformation $\mathrm{T}$ minimizes the distance between the sets $\left\{H_{i}\right\}$ and $\left\{X_{i}\right\}$.

$$
\begin{aligned}
\operatorname{Min} E_{j}= & \sum_{i=1}^{N} \operatorname{dis}_{j}\left(X_{i}-X_{i-1}, H_{i}-H_{i-1}\right) \\
& +T \sum_{i=1}^{N} \operatorname{dis}_{j}\left(X_{i}, C_{i}\right)
\end{aligned}
$$

where $\operatorname{dis}(X, H)=\|X-H\|^{2}$. The extraction of the closest contour point is discussed in [8]. The minimization process of $E_{j}$ is iterated so that in the $\mathrm{j}$-th iteration the set of measured points is $H^{j}=T H^{j-1}$ and the set of points $X^{j}$ is determined from the new points $H^{j}$. The iteration process is continued until $T$ becomes negligible.

The rigid transformation $T$ consists of the rotation matrix $R$ and the translation vector $t$ as shown in (2), which can be solved with the quaternion method.

$$
H_{i+1}=R H_{i}+t=\left(\begin{array}{cc}
\cos \theta & -\sin \theta \\
\sin \theta & \cos \theta
\end{array}\right) H_{i}+\left(\begin{array}{l}
t_{x} \\
t_{y}
\end{array}\right)
$$

where $\theta$ refers to the rotation angle. $t_{x}$ and $t_{y}$ are the translation.

The affine transformation $T_{A}$ is used in this paper to solve the problem that ICCP with rigid transformation cannot reduce the scaling error in INS indication track. Defining scale factor $S$, the matching process of the indication track $H_{i}$ to the real track $L_{i}$ of each iterative can be performed with (3).

$$
L_{i}=S R H_{i}+t
$$

The least squares solution of this transformation can be given by solving the Procrustes problem [9]. The cost function can be written as:

$$
\begin{aligned}
& f(S, R, t)_{i}=\sum_{i=1}^{n}\left\|L_{i}-\left(S R H_{i}+t\right)\right\|^{2} \\
& =n\left\|L_{0}-\left(S R H_{0}+t\right)\right\|^{2}+\sum_{i=1}^{n}\left\|L_{i}^{\prime}-\left(S R H_{i}^{\prime}+t\right)\right\|^{2}
\end{aligned}
$$

where $L_{0}$ and $H_{0}$ denote the centroids of $L_{1}$ and $H_{\mathrm{i}}, L_{i}^{\prime}$ and $H_{\mathrm{i}}^{\prime}$ is the relative vectors relative to their centroids. Then normalize $L_{\mathrm{i}}$ and $H_{\mathrm{i}}$

$$
L_{s, i}=L_{i} / \sqrt{\sum L_{0}^{2}}, H_{s, i}=H_{i} / \sqrt{\sum H_{0}^{2}}
$$

Define the matrix $A=L_{s} H_{s}^{T}$, where $L_{s}=\left[L_{s, 1}\right.$ $\left.L_{s, 2} \ldots L_{s, n}\right]$ and $H_{s}=\left[\begin{array}{lll}H_{s, 1} & H_{s, 2} \ldots H_{s, n}\end{array}\right]$. The least squares estimate of the rotation matrix $R$ is (6) when $A$ is nonsingular.

$$
R=A\left(A^{T} A\right)^{-1 / 2}
$$

Regarding the singular value decomposition of $A$ as $U \Lambda V^{T}$, the optimal rotation matrix $R$ can be expressed as: 


$$
R=V\left[\begin{array}{ccc}
1 & 0 & 0 \\
0 & 1 & 0 \\
0 & 0 & \operatorname{det}(U) \operatorname{det}(V)
\end{array}\right] U^{T}
$$

The scale factor $S$ is

$$
S=\operatorname{tr}(\Lambda) \cdot \sqrt{\sum L_{0}^{2}} / \sqrt{\sum H_{0}^{2}}
$$

Then minimize the first term contribution in Equation (4) by

$$
t=L_{0}-S R H_{0}
$$

\subsection{Information fusion}

According to positioning requirements, the system variables are defined as follows:

$$
\begin{gathered}
X(t)=\left[\phi_{E}, \phi_{N}, \phi_{U}, \delta V_{E}, \delta V_{N}, \delta L,\right. \\
\left.\delta \lambda, \varepsilon_{E}, \varepsilon_{N}, \varepsilon_{U}, \nabla_{E}, \nabla_{N}, \nabla_{U}\right]^{T}
\end{gathered}
$$

where $\phi_{E}, \phi_{N}, \phi_{U}$ is attitude misalignment angles in the direction, $\delta V_{E}, \delta V_{N}$ is velocity error in the east and north, $\delta L, \delta \lambda$ is the error of latitude and longitude, $\varepsilon_{E}, \varepsilon_{N}, \varepsilon_{U}$ is the drifts of the three gyroscopes, $\nabla_{E}, \nabla_{N}, \nabla_{U}$ is the drifts of the three accelerometers.

System state equation is followed as:

$$
\dot{X}(t)=F(t) X(t)+G(t) W(t)
$$

where $F(t)$ is System state matrix, $G(t)$ is System noise matrix and $W(t)$ is white noise in the gyros and accelerometers.

Measure equation is shown as:

$$
z(t)=h(t) x(t)+v(t)
$$

$z(t)$ is the differences of latitude and longitude between the INS and matching output. $h(t)$ is a zero matrix with dimension $2 \times 13$, except that $h(1,6)=1$ and $h(2,7)=1$. $v(t)$ is observation noise matrix, which is white noise.

\section{The INS Error Propagation Model}

The INS error propagation model actually used is more complex than the state equation in (11), and can be expressed as follows, with omitting the time variable $t$.

$$
\begin{gathered}
\dot{\boldsymbol{x}}=\boldsymbol{F}_{\text {INS }} \boldsymbol{x}+\boldsymbol{w} \\
\boldsymbol{x}=\left[\begin{array}{lllllll}
\varphi & \delta v^{n} & \delta p & \varepsilon^{b} & \nabla^{b} & \delta K_{g} & \delta K_{a}
\end{array}\right]^{T}
\end{gathered}
$$

where $\boldsymbol{\varphi}=\left[\begin{array}{lll}\varphi_{E} & \varphi_{N} & \varphi_{U}\end{array}\right]^{T}$ is platform misalignment angles; $\quad \boldsymbol{\delta} \boldsymbol{v}^{n}=\left[\begin{array}{lll}\delta v_{E}^{n} & \delta v_{N}^{n} & \delta v_{U}^{n}\end{array}\right]^{T}$ is velocity errors; $\boldsymbol{\delta} \boldsymbol{p}=\left[\begin{array}{lll}\delta L & \delta \lambda & \delta h\end{array}\right]^{T}$ represents latitude, longitude and altitude errors respectively; $\varepsilon^{b}=\left[\begin{array}{lll}\varepsilon_{x}^{b} & \varepsilon_{y}^{b} & \varepsilon_{z}^{b}\end{array}\right]^{T}$ is the gyro drift errors expressed in body frame; $\nabla^{b}=\left[\begin{array}{lll}\nabla_{x}^{b} & \nabla_{y}^{b} & \nabla_{z}^{b}\end{array}\right]^{T}$ is the accelerometer biases expressed in body frame;

$$
\begin{gathered}
\delta \boldsymbol{K}_{g}=\left[\begin{array}{lllll}
\delta k_{g x x} & \delta k_{g y x} & \delta k_{g z x} & \delta k_{g x y} & \delta k_{g y y} \\
& \delta k_{g z y} & \delta k_{g x z} & \delta k_{g y z} & \delta k_{g z z}
\end{array}\right]^{T}
\end{gathered}
$$

$$
\boldsymbol{\delta} \boldsymbol{K}_{a}=\left[\begin{array}{llllll}
\delta k_{a x x} & \delta k_{a y x} & \delta k_{a z x} & \delta k_{a y y} & \delta k_{a z y} & \delta k_{a z z}
\end{array}\right]^{T}
$$

and $\delta k_{g i i}, i=x, y, z$ are gyro scale factor errors; $\delta k_{g i j},(i, j=x, y, z, i \neq j) \quad$ are gyro actual axis misalignment angles with respect to ideal body frame axis; $\delta k_{a i i},(i=x, y, z)$ are accelerometer scale factor errors; $\delta k_{a i j},(i, j=x, y, z, i \neq j)$ are accelerometer actual axis misalignment angles with respect to ideal body frame axis. The components $\boldsymbol{\delta} \boldsymbol{K}_{g}, \boldsymbol{\delta} \boldsymbol{K}_{a}$ are all assumed to be constant vectors. $\boldsymbol{w}$ are white Gaussian noise sources including the first-order Gauss-Markov bias process for the accelerometer and gyro.

The $\boldsymbol{F}_{I N S}$ in (13) is expressed as:

$$
\boldsymbol{F}_{I N S}=\left[\begin{array}{ccccccc}
M_{a a} & M_{a v} & M_{a p} & -C_{b}^{n} & 0_{3 \times 3} & M_{a g} & 0_{3 \times 6} \\
M_{v a} & M_{v v} & M_{v p} & 0_{3 \times 3} & C_{b}^{n} & 0_{3 \times 9} & M_{v f} \\
0_{3 \times 3} & M_{p v} & M_{p p} & 0_{3 \times 3} & 0_{3 \times 3} & 0_{3 \times 9} & 0_{3 \times 6} \\
& & & 0_{21 \times 30} & & &
\end{array}\right]
$$

Assume the gyro sensed angular rate and accelerometer sensed specific force are $\omega_{i b}^{b}=\left[\begin{array}{lll}\omega_{i b x}^{b} & \omega_{i b y}^{b} & \omega_{i b z}^{b}\end{array}\right]$ and $f_{s f}^{b}=\left[\begin{array}{lll}f_{s f x}^{b} & f_{s f y}^{b} & f_{s f z}^{b}\end{array}\right]$, then the components in (15) are given as below:

$$
\begin{gathered}
\boldsymbol{M}_{a a}=\left(-\boldsymbol{\omega}_{i n}^{n} \times\right) \\
\boldsymbol{M}_{a v}=\left[\begin{array}{ccc}
0 & -1 / R_{M h} & 0 \\
1 / R_{N h} & 0 & 0 \\
\tan L / R_{N h} & 0 & 0
\end{array}\right]
\end{gathered}
$$

$$
\begin{aligned}
& \boldsymbol{M}_{a p}=\left[\begin{array}{ccc}
0 & 0 & v_{N}^{n} / R_{M h}^{2} \\
-\omega_{i e} \sin L & 0 & -v_{E}^{n} / R_{N h}^{2} \\
v_{E}^{n} \sec ^{2} L / R_{N h}+\omega_{i e} \cos L & 0 & -v_{E}^{n} \tan L / R_{N h}^{2}
\end{array}\right] \\
& M_{a g}=-\left[\begin{array}{lll}
\omega_{i b x}^{b} C_{b}^{n} & \omega_{i b y}^{b} C_{b}^{n} & \omega_{i b z}^{b} C_{b}^{n}
\end{array}\right] \\
& \boldsymbol{M}_{v a}=\left(\boldsymbol{f}_{s f}^{n} \times\right) \\
& \boldsymbol{M}_{v v}=\left(v^{n} \times\right) \boldsymbol{M}_{a v}-\left(\left(2 \omega_{i e}^{n}+\boldsymbol{\omega}_{e n}^{n}\right) \times\right)
\end{aligned}
$$




$$
\begin{aligned}
\boldsymbol{M}_{v p}=\left(v^{n} \times\right) & {\left[\begin{array}{ccc}
0 & 0 & v_{N}^{n} / R_{M h}^{2} \\
-2 \omega_{i e} \sin L & 0 & -v_{E}^{n} / R_{N h}^{2} \\
v_{E}^{n} \sec ^{2} L / R_{N h}+2 \omega_{i e} \cos L & 0 & -v_{E}^{n} \tan L / R_{N h}^{2} \\
0 & 0 & 0 \\
0 & 0 & 0 \\
-g_{0} \beta_{1} \sin L \cos L & 0 & \beta_{3}
\end{array}\right] }
\end{aligned}
$$

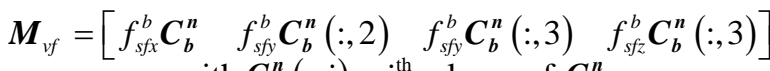$$
\text { with } \boldsymbol{C}_{b}^{n}(:, i)=i^{\text {th }} \text { column of } \boldsymbol{C}_{b}^{n}
$$$$
\boldsymbol{M}_{p v}=\left[\begin{array}{ccc}
0 & 1 / R_{M h} & 0 \\
\sec L / R_{N h} & 0 & 0 \\
0 & 0 & 1
\end{array}\right]
$$$$
\boldsymbol{M}_{p p}=\left[\begin{array}{ccc}
0 & 0 & -v_{N}^{n} / R_{M h}^{2} \\
v_{E}^{n} \sec L \tan L / R_{N h} & 0 & -v_{E}^{n} \sec L / R_{N h}^{2} \\
0 & 0 & 0
\end{array}\right]
$$

The " $\times$ " in $\omega_{i n}^{n} \times, f_{s f}^{n} \times, v^{n} \times$, etc. is the skewsymmetric operator of the vector, and where $f_{s f}^{n}=C_{b}^{n} f_{s f}^{b}$,

$$
\begin{aligned}
& \omega_{n b}^{b}=\omega_{i b}^{b}-C_{b}^{n}{ }^{T} \omega_{i n}^{n} \text {, and } \omega_{i n}^{n}=\omega_{i e}^{n}+\omega_{e n}^{n} \text {, with } \\
& \left\{\begin{array}{c}
\boldsymbol{\omega}_{i e}^{n}=\left[\begin{array}{lll}
0 & \omega_{i e} \cos L & \omega_{i e} \sin L
\end{array}\right]^{T} \\
\boldsymbol{\omega}_{e n}^{n}=\left[\begin{array}{lll}
-\frac{v_{N}^{n}}{R_{M h}} & \frac{v_{E}^{n}}{R_{N h}} & \frac{v_{E}^{n}}{R_{N h}} \tan L
\end{array}\right]^{T}
\end{array}\right. \\
& \left\{\begin{array}{c}
R_{M h}=R_{M}+h, R_{N h}=R_{N}+h \\
R_{M}=\frac{R_{N}\left(1-e^{2}\right)}{\left(1-e^{2} \sin ^{2} L\right)}, R_{N}=\frac{R_{e}}{\left(1-e^{2} \sin ^{2} L\right)^{1 / 2}} \\
e=\sqrt{2 f-f^{2}}
\end{array}\right. \\
& \left\{\begin{array}{c}
g^{n}=\left[\begin{array}{lll}
0 & 0 & -g
\end{array}\right]^{T} \\
g=g_{0}\left(1+\beta_{1} \sin ^{2} L+\beta_{2} \sin ^{4} L\right)-\beta_{3} h,
\end{array}\right. \\
& \text { with }\left\{\begin{array}{c}
\beta_{1}=5.27094 \times 10^{-3} \\
\beta_{2}=2.32718 \times 10^{-5} \\
\beta_{3}=2 g_{0} / R_{e}=3.086 \times 10^{-6}\left(1 / \mathrm{s}^{2}\right)
\end{array}\right.
\end{aligned}
$$

$C_{b}^{n}$ is transformation direct cosine matrix from body frame to navigation frame; $\boldsymbol{v}^{n}=\left[\begin{array}{lll}v_{E}^{n} & v_{N}^{n} & v_{U}^{n}\end{array}\right]^{T}$ is velocity along east, north and up-vertical direction; $\boldsymbol{p}=\left[\begin{array}{lll}L & \lambda & h\end{array}\right]^{T}$, where $L, \lambda, h$ is latitude, longitude and altitude; $R_{e}=6371.2 \mathrm{~km}$ is the Earth's semi-major axis; $f=1 / 298.257$ is the Earth's flattening; $\omega_{i e}=7.2921151467 ? 10^{5} \mathrm{rad} / \mathrm{s}$ is the Earth's angular rate; $g_{0}=9.7803267714 \mathrm{~m} / \mathrm{s}^{2}$ is gravity magnitude at the equatorial sea-surface.

\section{Simulation}

The effectiveness of the proposed matching algorithm and data fusion algorithm can be examined by this numerical simulation. The reference matching maps are generated with Enhanced Magnetic Model of order 720 and disturbed by Gaussian noise. The simulated magnetic measurement data are acquired based on the geomagnetic model above and disturbed by certain level external interferences, as shown in Table 1.

According to the simulation results in figure 3 , the traditional ICCP algorithm cannot effectively rectify the indication track to the real track, and also cannot get the correct matching results when there is a scaling error between the INS indication track and the real track. However, the improved algorithm achieves accurate matching results. At the same time, the improved ICCP algorithm can also effectively estimated gyro and accelerometer error as shown in Figure 4. The improved algorithm not only solves the scaling problem in the indication track during the geomagnetic matching process, but also accurately correct gyro drift and accelerometer error of INS.

Table 1. The parameters of simulation.

\begin{tabular}{cc}
\hline Parameter & Parameter Values \\
\hline Matching points & 50 \\
Reference map noise & $10 \mathrm{nT}$ \\
Matching region size & $10 \mathrm{~km} \times 8 \mathrm{~km}$ \\
Center location of matching region & $\left(108.4^{\circ} \mathrm{E}, 34.35^{\circ} \mathrm{N}\right)$ \\
Average altitude & $380 \mathrm{~m}$ \\
Gird size of matching region & $100 \times 80$ \\
Measurement Gaussian noise & $10 \mathrm{nT}$ \\
Matching Tolerance & $10 \mathrm{~m}$ \\
Platform misalignment angles & $1{ }^{\prime} / \mathrm{axis}$ \\
Initial velocity error & $10 \mathrm{~m} / \mathrm{s}, 30 \mathrm{~m} / \mathrm{s}$ \\
Initial position error & $-800 \mathrm{~m}, 1000 \mathrm{~m}$ \\
Gyro constant bias & $0.1 \mathrm{deg} / \mathrm{h}$ \\
Gyro random walk & $0.1 \mathrm{deg} / \mathrm{sqrt}(\mathrm{h})$ \\
Accelerometor constant bias & $50 \mu \mathrm{g}$ \\
Accelerometor random walk & $5 \mu \mathrm{g} / \mathrm{sqrt}(\mathrm{Hz})$ \\
Scale factor error & $10 \mathrm{ppm}$ \\
Askew installation error & $10^{\prime}$ \\
\hline
\end{tabular}

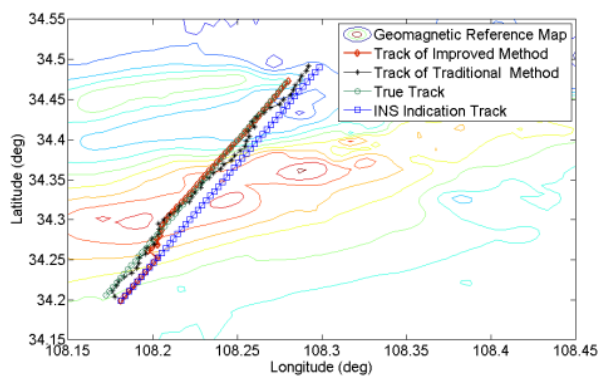

(a) 


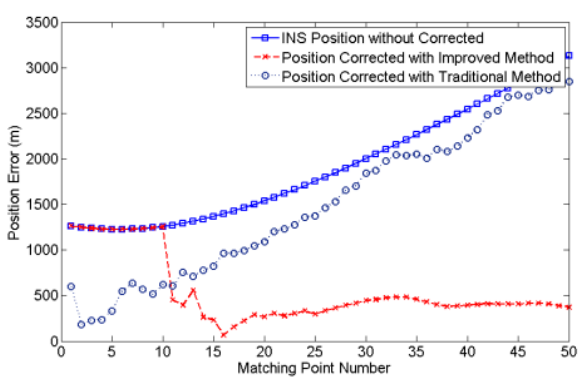

(b)

Figure 3. The comparison of the track and position error with proposed method and traditional method. The first ten points are not matched for collecting geomagnetic data.
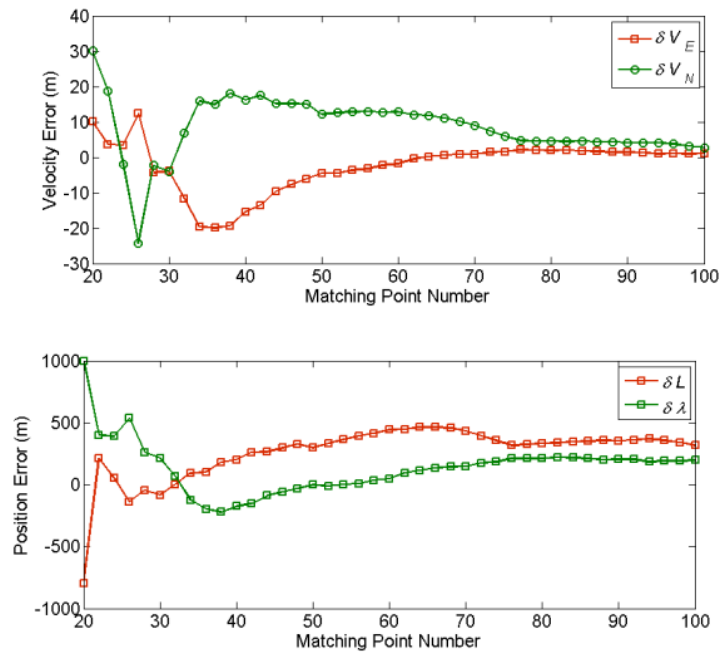

Figure 4. Convergence curve of INS

\section{Conclusion}

This paper proposed an improved method of geomagnetic aided inertial navigation algorithm with gyro and accelerometer error corrected, the affine transformation was adopted to ICCP to increase adaptability with indication track error characteristics of INS, and then the gyro drift and accelerometer deviation of INS were accurate estimated with EKF. The best achievable positioning accuracy was less than $300 \mathrm{~m}$ and velocity was less than $2 \mathrm{~m} / \mathrm{s}$ in case of existing scale error.

\section{Acknowledgment}

This work was supported in part by the National Natural Science Foundation of China (51707152), in part by the China Postdoctoral Science Foundation funded project (2014M562522XB), in part by the Shaanxi Province Natural Science Foundation of China (2016JQ4023), in part by the Scientific Research Program Funded by Shaanxi Provincial Education Department (15JK1523), and in part by the Scientific Research Project Foundation of Xi'an University of Technology (2016TS024).

\section{References}

1. F. Goldenberg, 2006 IEEE/ION Position, Location, And Navigation Symposium 14, 7 (2016)

2. H. Xin, X. Zhi, X. Jianxin and X. Limin, 2017 29th Chinese Control And Decision Conference (CCDC), (2016)

3. F. Haonan Feng, Y. Zhaohua, F. Jiancheng, 2008 2nd International Symposium on Systems and Control in Aerospace and Astronautics (2008)

4. Z. Yingying, Z. Yanshun and G. Lei, 2011 Third International Conference on Measuring Technology and Mechatronics Automation ( 2011)

5. Z. Dai and C. Kang, The 2014 2nd International Conference on Systems and Informatics (2014)

6. Y. Zhang, C. Li, S. Liu, M. Jiang and L. Guo, Proceedings of 2014 IEEE Chinese Guidance, Navigation and Control Conference (2014)

7. S. Zhongguo, Z. Jinsheng, Z. Wenqi, X. Xiaoli, Sensors, 7 (2016)

8. L. Byung-Uk, K. Chul-Min, P. Rae-Hong, IEEE Transactions on Pattern Analysis and Machine Intelligence, 22 (2000)

9. K. P.Behzad, K. P. Behrooz, Proceedings of SPIEThe International Society for Optical Engineering (1999)

10. L. Dorst, IEEE Trans. Pattern Anal. Mach. Intell, 27 (2005) 